\section{A inclusão de novas técnicas de análise citogenética aperfeiçoou o diagnóstico cromossômico da síndrome de Turner}

\author{
The inclusion of new techniques of chromosome analysis has \\ improved the cytogenetic profile of Turner syndrome
}

Beatriz Amstalden Barros' 1 Andréa Trevas Maciel-Guerra², Maricilda Palandi De Mello ${ }^{3}$, Fernanda Borchers Coeli ${ }^{3}$, Annelise Barreto de Carvalho', Nilma Viguetti-Campos², Juliana de Godoy Assumpção ${ }^{3}$, Antonia Paula Marques-de-Faria², Sofia Helena Valente de Lemos-Marini',Gil Guerra-Junior'

\section{RESUMO}

Objetivo: Avaliar o efeito do aprimoramento da análise cromossômica sobre os achados citogenéticos de pacientes com síndrome de Turner (ST). Métodos: Estudo retrospectivo dos resultados de cariótipo de 260 pacientes com ST, com análise das técnicas de bandamento, número de células avaliadas e pesquisa de sequências de cromossomoY. Segundo o cariótipo, dividiu-se em 45,X; mosaicismo cromossômico semY; aberrações estruturais de cromossomos sexuais com ou sem mosaicismo; mosaicismo com cromossomoY. Resultad os: $O$ cariótipo 45,X foi o mais frequente (108), seguido de aberrações estruturais (88) e mosaicismo (58 sem Y e 6 comY). A introdução de técnicas de bandamento e o aumento do número de células analisadas resultaram em redução progressiva de pacientes $45, \mathrm{X}$ e aumento de aberrações estruturais. $O$ estudo de sequências de cromossomo $Y$ foi feito em 96 casos e foi positivo em 10 . Conclusões: O aperfeiçoamento da análise cromossômica ao longo do tempo modificou o perfil citogenético da ST. Arq Bras Endocrinol Metab. 2009;53(9):1137-42

Descritores

Síndrome de Turner; cariótipo; técnicas citogenéticas; mosaicismo; cromossomo Y

\begin{abstract}
Objective: To evaluate the effect of the improvement of chromosome analysis on the cytogenetic findings of Turner syndrome (TS) patients. Methods: Retrospective study of the results of the karyotypes of 260 patients with TS, regarding banding techniques, number of cells analyzed and results of investigation of Y-chromosome sequences. According to karyotype, divided in $45, X$; sex chromosome mosaicism without $Y$; structural aberrations of sex chromosomes with or without mosaicism; sex chromosome mosaicism with $Y$. Results: $45, X$ was the most frequent karyotype (108), followed by structural aberrations (88) and mosaics (58 without $Y$ and 6 with $Y)$. Introduction of banding techniques and increase in the number of cells analyzed resulted in progressive decrease of $45, X$ karyotype and increase of structural aberrations. The study of $Y$ chromosome sequences was performed in 96 cases of which 10 resulted positive. Conclusions: Improvement of chromosome analysis over the years has modified the cytogenetic profile ofTS. Arq Bras Endocrinol Metab. 2009;53(9):1137-42
\end{abstract}

Keywords

Turner syndrome; karyotype; cytogenetic techniques; mosaicism; Y chromosome
'Departamento de Pediatria, Unidade de Endocrinologia Pediátrica, Faculdade de Ciências Médicas, Universidade Estadual de Campinas (FCMUnicamp), Campinas, SP, Brasil ${ }^{2}$ Departamento de Genética Médica, FCM-Unicamp, Campinas, SP, Brasil ${ }^{3}$ Centro de Biologia Molecular e Engenharia Genética (CBMEG), Unicamp, Campinas, SP, Brasil

Correspondência para: Gil Guerra-Júnior

Departamento de Pediatria, CP 6111 FCM-Unicamp

13083-970 - Campinas, SP, Brasil gilguer@fcm.unicamp.br

Recebido em 16/Mar/2009 Aceito em 25/Ago/2009 


\section{INTRODUÇÃO}

A síndrome de Turner (ST) ocorre pela perda parcial ou total de um dos cromossomos sexuais, sendo sua frequência estimada em 1:2.130 ou 0,47:1.000 nativivos do sexo feminino (1). Há uma alta seleção intrauterina contra a ST: por volta de $1 \%$ a $2 \%$ de todas as concepções humanas têm o cariótipo 45,X (2), porém 99\% delas evoluem para abortamento espontâneo (3).

Desde sua primeira descrição em 1938 até os anos 1990, o prognóstico das portadoras da ST foi considerado extremamente grave: quadro malformativo evidente, deficiência importante no crescimento e no desenvolvimento puberal, esterilidade, entre outros. Ainda hoje, para a maior parte dos médicos, a ST é considerada uma anomalia grave. No entanto, nos últimos 15 a 20 anos, novos conhecimentos, tanto no diagnóstico como na terapêutica para essas meninas, modificaram aquela visão inicial (4-6).

Os avanços terapêuticos foram principalmente no campo da promoção do crescimento com o uso do hormônio de crescimento recombinante humano (7), aperfeiçoamento das terapias de reposição hormonal para indução da puberdade (4-6) e técnicas de fertilização assistida para os casos de esterilidade $(5,6)$. Além disso, a pesquisa sistemática de anomalias congênitas, principalmente cardíacas e de vias urinárias, a investigação periódica de patologias adquiridas, especialmente as autoimunes, e a detecção citogenética e/ou molecular de sequências de cromossomo Y permitiram uma intervenção médica no sentido de evitar complicações (4-6).

O sucesso de toda essa abordagem depende, porém, fundamentalmente de um diagnóstico precoce e preciso da ST. As modificações que surgiram no decorrer desses últimos anos no diagnóstico da ST foram, inicialmente, de ordem conceitual. Quanto à baixa estatura e ao hipogonadismo primário, classicamente associados à ST, sabe-se hoje que não são características obrigatoriamente encontradas nessas pacientes (4-6). No que se refere às malformações, deve haver um continumm entre as anomalias tão graves que não permitem a sobrevivência do concepto até meninas e mulheres quase indistinguíveis daquelas da população normal (4-6). Além disso, tudo indica que o cariótipo classicamente associado à ST, o 45, X, não seja mais tão frequente, ao menos entre as nativivas (8-11).

Por isso o objetivo deste estudo foi avaliar a evolução do diagnóstico citogenético da ST desde o primeiro caso diagnosticado em 1970 no Laboratório de Citoge- nética Humana da Universidade Estadual de Campinas (Unicamp).

\section{CASUÍSTICA E MÉTODOS}

Foram avaliados retrospectivamente todos os casos com diagnóstico citogenético de ST, realizados pelo Laboratório de Citogenética Humana da Unicamp desde janeiro de 1970 até dezembro de 2008.

A avaliação consistiu do levantamento do resultado do cariótipo, do ano da realização do exame, do número de metáfases analisadas, e do resultado do estudo molecular de sequências do cromossomo Y. Todos os cariótipos avaliados foram realizados em cultura de linfócitos de sangue periférico e os resultados foram divididos em quatro grupos: $45, \mathrm{X}$; mosaicismos sem aberração estrutural de cromossomos sexuais e sem cromossomo Y; aberrações estruturais de cromossomos sexuais com ou sem mosaicismo; e mosaicismo com cromossomo Y íntegro. Os resultados em geral foram divididos em quatro etapas: primeira - de 1970 a 1980 - época em que a análise citogenética foi realizada por meio de coloração convencional, sem bandas, ou as técnicas de bandas eram incipientes, e frequentemente a contagem de metáfases não ultrapassava 16; segunda - de 1981 a 1988 - época em que a análise citogenética foi realizada rotineiramente por técnica de bandas $\mathrm{G}$ ou $\mathrm{Q}$, mas o número de metáfases analisadas variava entre 16 e 32; terceira - de 1989 a 1994 - após a criação do Grupo Interdisciplinar de Estudos da Determinação e Diferenciação do sexo (GIEDDS), com aumento do número de casos avaliados e com o aumento da contagem de metáfases para mais que 32 , frequentemente em 50; e quarta - a partir de 1995 - época em que foi incluída na avaliação dos casos de ST a pesquisa molecular por meio de hibridização com sondas específicas para as sequências de DNA do cromossomo Y (SRY, TSPY e DYZ3) (12) $\mathrm{e}$, dois anos depois, a presença de marcadores moleculares do cromossomo $\mathrm{Y}$ foi investigada primeiramente pela amplificação do gene $S R \Upsilon$ com os primers descritos na tabela 1; a presença do gene TSPY (Testis-Specific Protein $\Upsilon$-encoded) e da região centromérica DYZ3 foi verificada por nested-PCR utilizando primers externos e internos específicos (Tabela 1 ). Este estudo foi indicado nos casos com: a) cariótipo 45, X e cromatina X negativa; b) presença no cariótipo de cromossomos "marcadores", ou seja, fragmentos cromossômicos de origem indefinida; c) impossibilidade da identificação citogenética da origem de cromossomos em anel. 


\begin{tabular}{|c|c|c|c|}
\hline Gene & Primer direto $5^{\prime} \rightarrow 3^{\prime}$ & Primer reverso $5^{\prime} \rightarrow 3^{\prime}$ & Tamanho (pb) \\
\hline SRY & $\begin{array}{l}\text { CAT TGT CGA CCA GTG TGT } \\
\text { TGA AAC GGG AGA AAA CA }\end{array}$ & CAT TGT CGA CGT ACA ACC CTG TTG TCC AGT TGC & 380 \\
\hline TSPY JA 56/52 & CAT GAC ATA ATG GCG GAG & CGA TAG GCC TCC ACT TCA TA & 1300 \\
\hline TSPY JA 35/30* & CCC CAC CTA GAC CGC AGA GG & GGG AAG AAG GCC TAA GAG CAC C & 608 \\
\hline$D Y Z 3 A / B$ & TGA AAA CTA CAC AG- AAG CTG & ACA CAT CAC AAA GAA CTA TG & 1100 \\
\hline$D Y Z 3 \mathrm{G} / \mathrm{H}^{*}$ & AGC CTT TTG TGG CCT ACG & ATC CTC CTG GAG ATA CCA & 330 \\
\hline
\end{tabular}

* Primers internos para os loci TSPYe DYZ3 na nested-PCR.

O estudo foi aprovado pelo Comitê de Ética em Pesquisa da Faculdade de Ciências Médicas da Unicamp com parecer $\mathrm{n}^{\circ} 434 / 2006$.

Os dados foram apresentados em tabelas com valores absolutos e em percentagem e na análise estatística foi utilizado o teste de qui-quadrado com nível de significância abaixo de 5\%.

\section{RESULTADOS}

Das 260 pacientes com diagnóstico citogenético de ST de 1970 a $2008,19(7,3 \%)$ foram diagnosticadas de 1970 a 1980 ( 1,7 caso em média por ano), 41 ( $15,8 \%)$, de 1981 a 1988 (5,1 casos em média por ano); 200 $(76,9 \%)$, de 1989 a 2008 (10 casos em média por ano), observando-se evidente aumento do número de casos diagnosticados no decorrer dos anos.

Entre os 260 casos predominaram os cariótipos 45, X $(108=41,5 \%)$, seguidos pelas aberrações estruturais com ou sem mosaicismo $(88=33,8 \%)$, pelos mosaicos sem aberração estrutural e sem cromossomo Y $(58=22,3 \%)$ e pelos mosaicos com cromossomo $\mathrm{Y}$ íntegro $(6$ casos $=$ $2,3 \%$ ) (Tabela 2). Entre as aberrações estruturais, predominaram o isocromossomo de braço longo do cromosso$\operatorname{mo} \mathrm{X}$ [18 $\operatorname{casos} 46, \mathrm{X}, \mathrm{i}(\mathrm{X})(\mathrm{q} 10), 27 \operatorname{casos} 45, \mathrm{X} / 46, \mathrm{X}, \mathrm{i}(\mathrm{X})$ (q10) e mais 4 com cariótipos diversos] e os cromossomos "marcadores" (24 casos $45, \mathrm{X} / 46, \mathrm{X},+$ mar e mais 3 com cariótipos diversos) (Tabela 2).

Em relação à análise do número de metáfases, em 39 foram analisadas apenas 16 metáfases, em 45 a contagem variou de 17 a 32 (em geral 32 ) e em 176 foi superior a 32 (em geral 50 ).

Além dos 6 casos de cromossomo $\mathrm{Y}$ íntegro, o estudo de marcadores moleculares do cromossomo $\mathrm{Y}$ foi realizado em 96 pacientes, sendo positivo em 10: $5 / 20$ casos $45, \mathrm{X} / 46, \mathrm{X},+$ mar em que essa pesquisa foi realizada; $3 / 7045, \mathrm{X}$ avaliados e $2 / 3 \mathrm{pa}$ cientes com cariótipo 45,X/46,X,r(?). Entre os casos em que a pesquisa foi negativa, estão aqueles com cariótipo 45,X/46,X,i $(\mathrm{X})(\mathrm{ql} 10) / 46, \mathrm{X},+\mathrm{mar} / 47, \mathrm{X}, \mathrm{i}(\mathrm{X})$ (q10), +mar; 45,X/46,X,+mar/47,XX,+mar; e 45,X/ $46, \mathrm{XX} / 47, \mathrm{XX},+$ mar.

Com o passar dos anos, observaram-se diminuição progressiva na frequência de cariótipos $45, \mathrm{X}$, exceção da última etapa, e aumento na frequência de aberrações estruturais. Os mosaicismos sem aberração estrutural mantiveram uma frequência similar em todas as etapas. Em relação aos 45 , $\mathrm{X}$, observou-se diferença significativamente maior para as aberrações estruturais $\left(\chi_{(2)}^{2}=\right.$

\begin{tabular}{|c|c|}
\hline Cariótipo & $\mathbf{n}$ \\
\hline $45, X$ & 108 \\
\hline Subtotal & 108 \\
\hline \multicolumn{2}{|l|}{ Mosaicos sem aberração estrutural } \\
\hline $45, X / 46, X X$ & 50 \\
\hline $45, X / 47, X X X$ & 1 \\
\hline $45, X / 47, X X X / 46, X X$ & 7 \\
\hline Subtotal & 58 \\
\hline \multicolumn{2}{|l|}{ Mosaicos com aberração estrutural } \\
\hline $46, X, i(X)(q 10)$ & 18 \\
\hline $45, X / 46, X, i(X)(q 10)$ & 27 \\
\hline $45, X / 46, X, i(X)(q 10) / 46, X X$ & 2 \\
\hline $45, X / 46, X, i(X)(q 10) / 47, X, i(X)(q 10), i(X)(q 10)$ & 1 \\
\hline $45, X / 46, X, i(X)(q 10) / 46, X,+m a r / 47, X, i(X)(q 10),+$ mar & 1 \\
\hline $46, X, \operatorname{del}(X)(p)$ & 3 \\
\hline $45, X / 46, X, r(X)$ & 5 \\
\hline $45, X / 46, X, r(?)$ & 3 \\
\hline $45, X / 46, X, r(X) / 46, X X$ & 1 \\
\hline 45,X/46,X,del(X)(q?) & 1 \\
\hline $45, \mathrm{X} / 46, \mathrm{X},+\mathrm{mar}$ & 24 \\
\hline $45, \mathrm{X} / 46, \mathrm{X},+\mathrm{mar} / 47, \mathrm{XX},+\mathrm{mar}$ & 1 \\
\hline $45, \mathrm{X} / 46, \mathrm{XX},+\mathrm{mar} / 46, \mathrm{XX}$ & 1 \\
\hline Subtotal & 88 \\
\hline \multicolumn{2}{|l|}{ Com Y íntegro } \\
\hline $45, X / 46, X Y$ & 5 \\
\hline $45, X / 47, X Y Y$ & 1 \\
\hline Subtotal & 6 \\
\hline Total & 260 \\
\hline
\end{tabular}


14,34; $\mathrm{p}=0,0008)$, o mesmo não ocorrendo em relação aos mosaicos sem aberração estrutural $\left(\chi_{(2)}^{2}=0,70\right.$; $\mathrm{p}=0,74)($ Tabela 3$)$.

Quando se comparou a extensão da análise do número de metáfases com o resultado do cariótipo, verificou-se que em 27 das 108 pacientes com cariótipo 45,X (25\%) esse diagnóstico foi feito por meio da análise de um pequeno número de metáfases $(\leq 16)$, o mesmo não ocorrendo para os mosaicos sem aberração estrutural $(8 / 58=14 \%)$ e para as aberrações estruturais com ou sem mosaicismo $(4 / 88=4 \%)$ (Tabela 4$)$. Observou-se diferença significativamente maior dos mosaicos sem aberração estrutural para os $45, \mathrm{X}\left(\chi_{(2)}^{2}\right.$ $=7,73 ; \mathrm{p}=0,02)$ e das aberrações estrututurais com os $45, \mathrm{X}\left(\chi_{(2)}^{2}=14,59 ; \mathrm{p}=0,0007\right)$. Observaram-se também aumento do número de casos com mosaicismo e aberração estrutural associados com o passar dos anos e aumento da contagem de metáfases (Tabela 4).

\section{DISCUSSÃO}

Nos últimos anos, vem sendo progressivamente desmontada uma imagem estereotipada da ST: já não há mais cariótipo clássico, apesar de o 45, $\mathrm{X}$ ainda ser o mais frequente, nem fenótipo clássico, e o diagnóstico nem sempre salta aos olhos, mas sim tem de ser buscado ativamente, tanto do ponto de vista clínico quanto citogenético. Um dos sinais mais importantes nessa busca é o déficit de crescimento, manifestação precoce que deveria permitir um diagnóstico também precoce para que essas meninas venham a se beneficiar de várias medidas profiláticas e terapêuticas (4-7).

Viguetti e Maciel-Guerra (11) mostraram que uma em cada oito meninas, com baixa estatura e bom desenvolvimento neuropsicomotor, independente da presença de sinais dismórficos, encaminhadas ao Ambulatório de Pediatria do Hospital de Clínicas, apresentaram diagnóstico citogenético de ST, confirmando a importância da busca desse diagnóstico na baixa estatura em meninas. Lam e cols. (13), analisando retrospectivamente o cariótipo de 353 crianças de ambos os sexos com baixa estatura, encontraram 18,9\% de meninas com ST; enquanto Carvalho (14), avaliando retrospectivamente o cariótipo de 425 mulheres encaminhadas por baixa estatura e/ou puberdade atrasada e/ou amenorreia e/ou infertilidade, encontrou $36,9 \%$ de casos confirmados de ST.

O diagnóstico citogenético da ST vem evoluindo e modificando-se substancialmente ao longo dos anos.

\begin{tabular}{|c|c|c|c|c|c|c|c|c|c|}
\hline \multirow[b]{3}{*}{ Época } & \multicolumn{9}{|c|}{ Cariótipo } \\
\hline & \multicolumn{2}{|c|}{$45, X$} & \multicolumn{2}{|c|}{$\begin{array}{c}\text { Mosaicos sem } \\
\text { aberração } \\
\text { estrutural }\end{array}$} & \multicolumn{2}{|c|}{$\begin{array}{l}\text { Aberrações } \\
\text { estruturais }\end{array}$} & \multicolumn{2}{|c|}{$Y$ íntegro } & \multirow{2}{*}{$\begin{array}{r}\text { Tota } \\
\mathbf{n}\end{array}$} \\
\hline & $n$ & $\%$ & $\mathrm{n}$ & $\%$ & $\mathbf{n}$ & $\%$ & $\mathbf{n}$ & $\%$ & \\
\hline 1970 a 1980 & 12 & 63 & 7 & 37 & 0 & 0 & 0 & 0 & 19 \\
\hline 1981 a 1988 & 20 & 49 & 8 & 19 & 9 & 22 & 4 & 10 & 41 \\
\hline 1989 a 2008 & 76 & 38 & 43 & 21 & 79 & 40 & 2 & 1 & 200 \\
\hline Total & 108 & & 58 & & 88 & & 6 & & 260 \\
\hline
\end{tabular}

\begin{tabular}{|c|c|c|c|c|c|c|c|c|c|c|c|c|}
\hline & \multicolumn{12}{|c|}{ Cariótipo } \\
\hline & \multicolumn{3}{|c|}{$45, X$} & \multicolumn{3}{|c|}{$\begin{array}{l}\text { Mosaico sem aberração } \\
\text { estrutural }\end{array}$} & \multicolumn{3}{|c|}{ Aberrações estruturais } & \multicolumn{3}{|c|}{ Y íntegro } \\
\hline & $\begin{array}{l}1970- \\
1980\end{array}$ & $\begin{array}{l}1981- \\
1988\end{array}$ & $\begin{array}{l}1989- \\
2008\end{array}$ & $\begin{array}{l}1970- \\
1980\end{array}$ & $\begin{array}{l}1981- \\
1988\end{array}$ & $\begin{array}{l}1989- \\
2008\end{array}$ & $\begin{array}{l}1970- \\
1980\end{array}$ & $\begin{array}{l}1981- \\
1988\end{array}$ & $\begin{array}{l}1989- \\
2008\end{array}$ & $\begin{array}{l}1970- \\
1980\end{array}$ & $\begin{array}{l}1981- \\
1988\end{array}$ & $\begin{array}{l}1989- \\
2008\end{array}$ \\
\hline Metáfases* & $n$ & $n$ & $n$ & $n$ & $n$ & $n$ & $n$ & $n$ & $n$ & $n$ & $n$ & $n$ \\
\hline $12(8-16)$ & 12 & 15 & 0 & 7 & 1 & 0 & 0 & 3 & 1 & 0 & 0 & 0 \\
\hline $26(20-32)$ & 0 & 3 & 6 & 0 & 4 & 3 & 0 & 4 & 19 & 0 & 4 & 2 \\
\hline $65(50-100)$ & 0 & 2 & 70 & 0 & 3 & 40 & 0 & 2 & 59 & 0 & 0 & 0 \\
\hline Total & 12 & 20 & 76 & 7 & 8 & 43 & 0 & 9 & 79 & 0 & 4 & 2 \\
\hline
\end{tabular}

* Mediana (variação) do número de metáfases analisadas. 
Inicialmente, a modificação veio como resposta à implantação efetiva das técnicas de bandamento cromossômico. "Surgiram", a partir de então, as aberrações estruturais, com diminuição dos casos 45,X. De $1989 \mathrm{em}$ diante, porém, observou-se nova queda na frequência de cariótipos $45, \mathrm{X}$, associada a um aumento na frequência de aberrações estruturais. A explicação para esse fato, porém, é mais difícil de ser encontrada. Isso porque, a partir dessa data, foram introduzidas duas modificações simultâneas: a pesquisa sistemática de meninas com baixa estatura, atendidas no Ambulatório de Pediatria do Hospital de Clínicas, independente de quão grave fosse o déficit de crescimento e da presença de sinais dismórficos, e o aumento do número de metáfases analisadas por cariótipo. Com o decorrer dos anos, a investigação de ST em nosso serviço tem ocorrido em crianças com estatura baixa, porém menos comprometida, o que pode ser observado na análise dos dados de duas teses ocorridas nesse período, porém ainda sem modificação da faixa etária ao diagnóstico $(14,15)$ (Tabela 5).

Tabela 5. Estatura (em escore z) e idade (em anos) das pacientes com síndrome de Turner diagnosticadas pelo Laboratório de Citogenética Humana da Unicamp de 1970 a 2006

\begin{tabular}{lccc}
\hline \multirow{2}{*}{ Ano } & n & Idade (anos) & Estatura (escore z) \\
\cline { 3 - 4 } & & $\begin{array}{c}\text { Média } \pm \text { DP } \\
\text { (mínimo a máximo) }\end{array}$ & $\begin{array}{c}\text { Média } \pm \text { DP } \\
\text { (mínimo a máximo) }\end{array}$ \\
\hline $1970-1988^{15}$ & 60 & $13,1 \pm 7,1$ & $-4,17 \pm 1,45$ \\
& & $(0,1 \mathrm{a} 31,8)$ & $(-7,76 \mathrm{a}-1,06)$ \\
$1989-2006^{14}$ & \multirow{2}{*}{155} & $12,4 \pm 7,3$ & $-3,09 \pm 1,34$ \\
& & $(0,1 \mathrm{a} 33,8)$ & $(-8,79 \mathrm{a} 0,16)$ \\
\hline
\end{tabular}

Ambas as modificações foram significativas: as pacientes são atualmente menos baixas, e os laudos de cariótipo $45, \mathrm{X}$, mais rigorosos, permitindo a detecção de mosaicismo em maior número de casos. O estudo de Held e cols. (8) mostrou que a simples extensão da análise citogenética a um segundo tecido reduz a frequência de cariótipos $45, \mathrm{X}$ para pouco mais de $21 \%$, enquanto Fernández e cols. (16) comprovaram que a avaliação de centenas ou milhares de metáfases por hibridização in situ com sondas específicas para o cromossomo $\mathrm{X}$ é capaz de reduzir a menos de $10 \%$ o número de casos em que o cariótipo permanece sendo $45, \mathrm{X}$, além de detectar outras linhagens celulares em pacientes que já tinham diagnóstico de mosaicismo pelo estudo citogenético convencional. $\mathrm{O}$ aumento no número de metáfases analisadas, de 11 a 16 para 50 , poderia, por- tanto, ter contribuído para a modificação nos achados citogenéticos.

A probabilidade de encontro de ao menos uma célula com uma constituição cromossômica diversa da 45,X depende, teoricamente, apenas da frequência das linhagens celulares em mosaico e do número de células analisadas (17). Viguetti-Campos (18) demonstrou, porém, em casos de mosaicismo 45,X/46,XX, que após 72 horas de cultura a proporção de núcleos interfásicos com um único sinal por hibridização in situ com sondas específicas para o cromossomo X (que devem corresponder às células $45, \mathrm{X})$ foi significativamente superior à proporção de metáfases $45, \mathrm{X}$ no cariótipo. Isso sugere que haja interferências no procedimento de análise cromossômica convencional que levem a modificações na proporção real de diferentes linhagens celulares, e que, portanto, esse não seja um procedimento aleatório. Nesse caso, as tabelas de cálculo de probabilidade de detecção de mosaicismo na dependência do número de células analisadas e da frequência das linhagens celulares (17) não refletiriam a realidade, e é possível que o aumento no número de metáfases analisadas observado neste trabalho tenha repercutido muito mais intensamente do que se poderia supor.

Mais difícil é determinar se houve influência da pesquisa sistemática de meninas com baixa estatura, independente de sua magnitude, sobre a queda na frequência de cariótipos 45 , $\mathrm{X}$ e consequente aumento das aberrações estruturais. Uma hipótese é a de que, entre as meninas com déficit de crescimento menos acentuado, fosse menos frequente o cariótipo 45, X e mais frequentes as aberrações estruturais. No entanto, recentemente, Lemos-Marini e cols. (19) não encontraram associação entre a altura final de 58 casos de ST com o resultado do cariótipo.

Outros estudos com grande número de casos avaliados e que buscaram correlações entre os diversos tipos de cariótipo e a estatura, como os de Pelz e cols. (20)e Ranke e cols. (21), não encontraram diferenças significativas no padrão da curva de crescimento e na estatura final. Levando-se em consideração os resultados desses trabalhos, a averiguação dos casos pela baixa estatura não teria tido qualquer interferência sobre a proporção dos diferentes cariótipos.

Outro resultado deste estudo mostra que a procura de sequências moleculares de cromossomo $\mathrm{Y}$, ou por qualquer outro método, como citogenética molecular (FISH: fluorescence in situ bybridization) em casos selecionados de ST (cariótipos com cromossomos mar- 
cadores e cariótipos com monossomia $45, \mathrm{X}$ em, no mínimo, 50 metáfases analisadas), está justificada pela frequência encontrada e pela associação com risco de neoplasia gonadal, como já mostrado por outros autores (22-25).

Portanto, pode-se concluir que os aprimoramentos diagnósticos ocorridos no Laboratório de Citogenética Humana da Unicamp, no decorrer desses últimos 39 anos, permitiram melhorar a qualidade do resultado citogenético da ST, com modificação da proporção entre os tipos de cariótipos observados.

Agradecimentos: ao Conselho Nacional de Desenvolvimento Científico e Tecnológico (CNPq) (GGJr - processo 500446/2007-5 bolsa de iniciação científica para $\mathrm{BAB}$ ), e à Fundação de Amparo à Pesquisa do Estado de São Paulo (Fapesp) (GGJr - processo 07/00520-3).

Declaração: os autores declaram não haver conflitos de interesse científico neste estudo.

\section{REFERÊNCIAS}

1. Nielsen J, Vohlert M. Sex chromosome abnormalities found among 34,910 newborn children: results from a 13-year incidence study in Arhus, Denmark. Birth Defects Orig Artic Ser. 1991;24:209-23.

2. De La Chapelle A. Sex chromosome abnormalities. In: Emey AEH, Rimon DL, editors. Principles and practice of medical genetics. Edinburgh: Churchill Livingstone; 1983. p. 193-215.

3. Hook EB, Warburton D. The distribution of chromosomal genotypes associated with Turner syndrome: livebirth prevalence rates and evidence for diminished fetal mortality and severity in genotypes associated with structural $\mathrm{X}$ abnormalities or mosaicism. Hum Genet. 1983;64:24-7.

4. Gravholt $\mathrm{CH}$. Epidemiological, endocrine and metabolic features in Turner syndrome. Eur J Endocrinol. 2004;151:657-87.

5. Gravholt $\mathrm{CH}$. Clinical practice in Turner syndrome. Nat Clin Pract Endocrinol Metab. 2005;1:41-52.

6. Hjerrild $\mathrm{BE}$, Mortensen $\mathrm{KH}$, Gravholt $\mathrm{CH}$. Turner syndrome and clinical treatment. Br Med Bull. 2008;86:77-93.

7. Baxter L, Bryant J, Cave CB, Milne R. Recombinant growth hormone for children and adolescents with Turner syndrome. Cochrane Database Syst Rev. 2007;24:CD003887.

8. Held KR, Kerber S, Kaminsky E, Singh S, Goetz P, Seemanova E, et al. Mosaicism in 45,XTurner syndrome: does survival in early pregnancy depend on the presence of two sex chromosomes? Hum Genet. 1992;88:288-94.
9. Rochiccioli P, Tauber MT. Le syndrome de Turner: une revolution. Arch Fr Pédiatr. 1992;49:153-5.

10. Uzielli MLG, Failli M, Biondi C. From chromosomes and genes to the discovery of Turner syndrome secrets. In: Hibi I, Takano K, editors. Basic and clinical approach to Turner syndrome. Amsterdam: Elsevier Science Publishers B.V.; 1993. p. 27-32.

11. Viguetti NL, Maciel-Guerra AT. Baixa estatura na infância e síndrome deTurner: uma associação mais freqüente do que se supõe. J Pediatr (Rio J). 1994;70:172-4.

12. Assumpção JG. Estudo de seqüências do cromossomo $Y$ em pacientes com distúrbios de diferenciação gonadal. Dissertação de Mestrado, Instituto de Biologia, Universidade Estadual de Campinas, 1998.

13. Lam WF, Hau WL, LamTS. Evaluation of referrals for genetic investigation of short stature in Hong Kong. Clin Med J. 2002;115:607-11.

14. Carvalho AB. Fatores associados ao encaminhamento de pacientes com suspeita clínica de síndrome de Turner a um serviço universitário de referência. Dissertação de Mestrado, Faculdade Ciências Médicas, Universidade Estadual de Campinas, 2009.

15. Maciel-Guerra AT. A evolução dos diagnósticos clínico e citogenético da síndrome de Turner: cherchez la petite fille. Tese de livredocência, Faculdade de Ciências Médicas, Universidade Estadual de Campinas, 1998.

16. Fernández R, Méndez J, Pássaro E. Turner syndrome: a study of chromosomal mosaicism. Hum Genet. 1996;98:29-35.

17. Beiguelman B. As cromossomopatias autossômicas. In: Citogenética humana. Rio de Janeiro: Guanabara Koogan; 1982. p. 179-218.

18. Viguetti-Campos NL. Estudo da seleção in vitro de linhagens $45, X$ por meio de hibridização in situ. Tese de doutorado, Instituto de Biologia, Universidade Estadual de Campinas, 1997.

19. Lemos-Marini SHV, Morcillo AM, Baptista MTM, Guerra-Junior G, Maciel-Guerra AT. Spontaneous final height in Turner's syndrome in Brazil. J Pediatr Endocrinol Metab. 2007;20:1207-14.

20. Pelz L, Timm D, Eyermann E, Hinkel GK, Kirchner M, Verron G. Body's height in Turner's syndrome. Clin Genet. 1982;22:62-6.

21. Ranke MB, Pfluger $H$, Rosendahl $W$, Stubbe $P$, Enders $H$, Bierich JR, et al. Turner syndrome: spontaneous growth in 150 cases and review of the literature. Eur J Pediatr. 1983;141:81-8.

22. Canto $P$, Kofman-Alfaro $S$, Jimenez AL, Soderlund D, Barron $C$, Reyes $\mathrm{E}$, et al. Gonadoblastoma in Turner syndrome patients with nonmosaic $45, X$ karyotype and $Y$ chromosome sequences. Cancer Genet Cytogenet. 2004;150:70-2.

23. Horn LC, Limbach A, Hoepffner W, Trobs RB, Keller E, Froster UG, et al. Histologic analysis of gonadal tissue in patients with UllrichTurner syndrome and derivativeY chromosomes. Pediatr Dev Pathol. 2005;8:197-203.

24. Mazzanti L, Cicognani A, Baldazzi L, Bergamaschi R, Scarano E, Strocchi S, et al. Gonadoblastoma in Turner syndrome and Ychromosome-derived material. Am J Med Genet. 2005;135:150-4.

25. Brant WO, Rajimwale A, Lovell MA, Travers SH, Furness PD 3rd, Sorensen $\mathrm{M}$, et al. Gonadoblastoma and Turner Syndrome. J Urol. 2006;175:1858-60. 